\title{
EpitopeVec: linear epitope prediction using deep protein sequence embeddings
}

\author{
Akash Bahai $\mathbb{1}^{1,2}$, Ehsaneddin Asgari $\mathbb{1}^{1,3}$, Mohammad R. K. Mofrad ${ }^{3,4}$, \\ Andreas Kloetgen ${ }^{1}$ and Alice C. McHardy ${ }^{1,2, *}$
}

\begin{abstract}
${ }^{1}$ Computational Biology of Infection Research, Helmholtz Center for Infection Research, Braunschweig 38124, Germany, ${ }^{2}$ Braunschweig Integrated Center of Systems Biology (BRICS), Technische Universität Braunschweig, Braunschweig 38106, Germany, ${ }^{3}$ Molecular Cell Biomechanics Laboratory, Department of Bioengineering and Mechanical Engineering, University of California, Berkeley, CA 94720, USA and ${ }^{4}$ Molecular Biophysics and Integrated Bioimaging, Lawrence Berkeley National Lab, Berkeley, CA 94720, USA
\end{abstract}

*To whom correspondence should be addressed.

Associate Editor: Jonathan Wren

Received on December 12, 2020; revised on May 28, 2021; editorial decision on June 17, 2021; accepted on June 25, 2021

\begin{abstract}
Motivation: B-cell epitopes (BCEs) play a pivotal role in the development of peptide vaccines, immuno-diagnostic reagents and antibody production, and thus in infectious disease prevention and diagnostics in general. Experimental methods used to determine BCEs are costly and time-consuming. Therefore, it is essential to develop computational methods for the rapid identification of BCEs. Although several computational methods have been developed for this task, generalizability is still a major concern, where cross-testing of the classifiers trained and tested on different datasets has revealed accuracies of $51-53 \%$.

Results: We describe a new method called EpitopeVec, which uses a combination of residue properties, modified antigenicity scales, and protein language model-based representations (protein vectors) as features of peptides for linear BCE predictions. Extensive benchmarking of EpitopeVec and other state-of-the-art methods for linear BCE prediction on several large and small datasets, as well as cross-testing, demonstrated an improvement in the performance of EpitopeVec over other methods in terms of accuracy and area under the curve. As the predictive performance depended on the species origin of the respective antigens (viral, bacterial and eukaryotic), we also trained our method on a large viral dataset to create a dedicated linear viral BCE predictor with improved cross-testing performance.
\end{abstract}

Availability and implementation: The software is available at https://github.com/hzi-bifo/epitope-prediction.

Contact: alice.mchardy@helmholtz-hzi.de

Supplementary information: Supplementary data are available at Bioinformatics online.

\section{Introduction}

Antibodies are critical components of the humoral immune response that recognize and bind to the antigens of pathogens, such as bacteria or viruses (Janeway, 2012). The region of an antigen recognized by these antibodies is known as an epitope and it can either be a continuous stretch of amino acids within an antigen protein sequence (linear epitope) or amino acids potentially separated in the sequence but located closely in the 3D protein structure (conformational epitope). In particular, the identification of B-cell epitopes (BCEs) is important for applications, such as peptide-based vaccine design (Dudek et al., 2010), immuno-diagnostic tests (Noya et al., 2005) and synthetic antibody production (Hancock and O'Reilly, 2005). As the experimental determination of BCEs is timeconsuming and expensive, computational prediction can play a pivotal role in the development of new vaccines and drugs against common viral pathogens such as human immunodeficiency virus, hepatitis or influenza viruses (Bryson et al., 2010; Dudek et al., 2010; Pellequer et al., 1991; Sanchez-Trincado et al., 2017). Although the majority of naturally occurring BCEs are conformational (Barlow et al., 1986), the prediction of linear BCEs has received much attention (Flower, 2007), as they are used for the synthesis of peptide-based vaccines among others (Malonis et al., 2020; Soria-Guerra et al., 2015).

The earliest methods for epitope prediction evaluated only one physiochemical property of the constituent amino acids, such as surface accessibility (Emini et al., 1985), flexibility (Karplus and Schulz, 1985), hydrophobicity (Levitt, 1976), or antigenicity (Kolaskar and Tongaonkar, 1990). Some of these methods that are still accessible online include PREDITOP (Pellequer and Westhof, 
Table 1. Summary of the datasets used in the benchmarking of our approach

\begin{tabular}{llccll}
\hline Dataset & $\begin{array}{l}\text { Original } \\
\text { method }\end{array}$ & Epitopes & $\begin{array}{l}\text { Non- } \\
\text { epitopes }\end{array}$ & Source & Length \\
\hline BCPreds & BCPreds & 701 & 701 & Bcipep & 20 \\
ABCPred16 & ABCPred & 700 & 700 & Bcipep & 16 \\
Chen & AAP & 872 & 872 & Bcipep & 20 \\
Blind387 & ABCPred & 187 & 200 & Various & Not fixed \\
LBTope_fixed_nr & LBTope & 7824 & 7853 & IEDB & 20 \\
iBCE-EL training & iBCE-EL & 4440 & 5485 & IEDB & Not fixed \\
iBCE-EL ind. & iBCE-EL & 1110 & 1408 & IEDB & Not fixed \\
Viral & New & 4432 & 8460 & IEDB & Not fixed \\
\hline
\end{tabular}

Note: The datasets are available at https://github.com/hzi-bifo/epitope-pre diction-paper.

1993), PEOPLE (Alix, 1999) and BEPITOPE (Odorico and Pellequer, 2003). These algorithms calculate the average amino acid propensity scale for individual features over a sliding window along the query protein sequence. If these predicted scales are above a certain cut-off for a continuous stretch of the protein, then the respective region on the sequence is determined to be a linear BCE. However, an assessment of 484 propensity scales revealed that such scales are unreliable for detecting BCEs and barely outperformed random BCE selection when based on a single amino acid feature or even a combination of features (Blythe and Flower, 2005).

With the increased availability of experimentally identified epitopes, new methods have been based on several propensity scales and included additional amino acid features that had not been included before (Yang and Yu, 2009). Such methods, which use machinelearning (ML) approaches to distinguish BCEs from non-BCEs in the amino acid sequence, have shown better accuracy than single propensity scale-based methods. For training the ML model, BCEs are presented as feature vectors derived from different amino acid properties, such as the amino acid composition (AAC), the amino acid pair (AAP) antigenicity scale (Chen et al., 2007) or the amino acid trimer (AAT) antigenicity scale (Yao et al., 2012). Some examples of ML-based methods for BCE prediction are BepiPred (Larsen et al., 2006), ABCPred (Saha and Raghava, 2006), LBTope (Singh et al., 2013), AAP (Chen et al., 2007), APCPred (Shen et al., 2015), BCPreds (El-Manzalawy et al., 2008), Cobe-Pro (Sweredoski and Baldi, 2009), SVMTrip (Yao et al., 2012), iBCE-El (Manavalan et al., 2018), BepiPred 2.0 (Jespersen et al., 2017), DLBEpitope (Liu et al., 2020) and EpiDope (Collatz et al., 2021) . A notable issue seems to be that all the aforementioned methods lack high accuracy in a cross-testing approach, where ML training and testing are performed on independent datasets, which raises doubts about the generalizability of all such approaches (Galanis et al., 2021; Sun et al., 2018).

\subsection{Contributions}

Here, we describe a new method that combines commonly used propensity scales, residue features and modified antigenicity scales with ProtVec (Asgari, 2019; Asgari and Mofrad, 2015; Asgari et al., $2019 \mathrm{~b})$ for vector representation of the peptides instead of the commonly used one-hot encoding method. Notably, EpiDope, a recently published study, also makes use of protein embeddings, but it uses context-aware embeddings for each residue in the sequence by using a vector with a length of 1000 , whereas ProtVec embeddings are context-independent and encode the entire sequence by using a vector with a length of 300 . Previously, sequence-based embeddings have been used successfully in functional-structural protein annotation tasks, such as predictions of secondary structures (Asgari et al., 2019a; $\mathrm{Li}$ and $\mathrm{Yu}, 2016$ ), the effects of mutations on protein-protein interactions (Zhou et al., 2020), protein functions (Asgari and Mofrad, 2015; Bonetta and Valentino, 2020; Zhou et al., 2019) and structural motifs (Liu et al., 2018). We used a support vector machine (SVM) with a Radial Basis Function (RBF) kernel as our predictive model. We trained and tested our method on multiple small and large datasets derived from the Bcipep (Saha et al., 2005) and Immune Epitope Database (IEDB) (Vita et al., 2010) and compared its performance with the state-of-the-art methods. We also cross-tested some of the methods (BepiPred, iBCE-EL, BepiPred2.0, DLBEpitope and EpiDope) on datasets on which they had not been tested before to verify the reliability and generalizability of these approaches. To establish a fair comparison, we trained our method on the same datasets on which the original methods were trained and tested the performance on the same test sets that the original methods used. Lastly, we trained our method on a large viral dataset in order to construct a predictor for linear viral BCEs as a resource available for the community.

\section{Materials and methods}

\subsection{Datasets}

Most of the methods published in the literature were trained on datasets compiled from databases of experimentally verified epitopes, such as Bcipep or IEDB. Earlier methods, such as ABCPred, BCPreds and AAP, were trained on datasets compiled from the Bcipep database. More recent methods, such as LBTope, SVMTrip, iBCE-El, BepiPred 2.0, DLBEpitope and EpiDope, were trained on datasets compiled from the IEDB. We compiled a list of benchmarking datasets and compared our method with the previously published studies using these datasets for a fair and comprehensive comparison (Table 1 and Supplementary Section 1).

\subsubsection{Viral dataset}

We downloaded viral peptides from the IEDB in which peptides were reported as epitopes (positive) and non-epitopes (negative). The peptide length was not fixed and it varied from 6 to 46 amino acids. We used CD-HIT (Huang et al., 2010) to remove homologous peptide sequences (cut-off: $80 \%$ for positive and $90 \%$ for negative sequences) in the dataset. As the same peptide can be reported to be an epitope and a non-epitope in different neutralization experiments, we removed the peptides that were common in both sets to finally obtain a dataset of 4432 peptides that were epitopes (positive) and 8460 peptides that were non-epitopes (negative).

\subsection{Feature representation of peptides}

\subsubsection{Amino acid composition}

The AAC is represented by a vector specifying the relative abundance of each amino acid in the peptide. It can be formulated as:

$$
A A C(P)=\left(f_{1}, f_{2}, f_{3}, \ldots, f_{20}\right),
$$

where $f_{i}=\frac{R_{i}}{N}(i=1,2, \ldots, 20)$ is the percentage comprised by amino acid type $i, R_{i}$ is the count of type $i$ in the peptide and $N$ is the peptide length.

\subsubsection{Dipeptide composition}

Dipeptide composition (DPC) is represented by a vector specifying the abundance of dipeptides normalized by all possible dipeptide combinations for a peptide $P$. It has a fixed length of $400(20 \times 20)$ features. It can be formulated as:

$$
\operatorname{DPC}(P)=\left(f_{1}, f_{2}, f_{3}, \ldots, f_{400}\right),
$$

where $f_{i}=\frac{R_{i}}{N}(i=1,2, \ldots, 400)$ is the percentage of composition of dipeptide type $i, R_{i}$ is the count of type $i$ in the peptide and $N$ is the peptide length.

\subsubsection{AAP antigenicity scale}

The AAP antigenicity scale was introduced by Chen et al. (2007). It is the ratio of how frequently AAPs occur in the positive set compared with the negative set. The antigenicity value for each dipeptide is the logarithm of the frequency in the positive set divided by the frequency in the negative set. We normalized the scale between +1 
and -1 to avoid the dominance of an individual propensity value. For the positive set, we used the Bcipep dataset; for the negative dataset, we chose the entire UniProt50 database from Swiss-Prot (Bairoch, 2000), which contains 561908 protein sequences:

$$
R_{A A P}=\log \left(\frac{f_{A A P}^{+}}{f_{A A P}^{-}}\right) .
$$

\subsubsection{AAT antigenicity scale}

The AAT antigenicity scale was first introduced in SVMTrip (Yao et al., 2012). It is similar to the AAP scale, except that it uses amino acid triplets instead of AAPs. The AAT scale is the logarithm of the ratio of the frequency of amino acid triplets in the positive set to their frequency in the negative set. The scale is normalized between +1 and -1 , similar to the AAP scale:

$$
R_{A A T}=\log \left(\frac{f_{A A T}^{+}}{f_{A A T}^{-}}\right) .
$$

\subsection{5 $K$-mer representation}

Segmentation of biological sequences into a bag of overlapping fixed-length $k$-mers is one of the most common representations in bioinformatics research. $K$-mer representations are widely used in the areas of proteomics (Grabherr et al., 2011), genomics (Alipanahi et al., 2015; Jolma et al., 2013), epigenomics (Awazu, 2017; Giancarlo et al., 2015) and metagenomics (Asgari et al., 2018; Wood and Salzberg, 2014). To create a $k$-mer representation of a given protein sequence, the sequences are divided into overlapping subsequences of length $k$ ( $k$-mers). Subsequently, they are represented as a frequency vector of all possible amino acid $k$-mers (vectorsize $=|20|^{k}$, where 20 is the number of amino acids).

\subsubsection{ProtVec sequence embeddings}

Recently, in natural language processing (NLP), continuous vector representations of words known as word embeddings have become a very popular approach for word representations in downstream ML tasks. The general idea is to learn a vector representation of words in the course of neural probabilistic language modeling, and then use the learned representation as a general-purpose representation of words in any NLP task. Language modeling is the task of assigning a probability to a given sequence of words or predicting the next word, given the previous words. There are two main reasons for choosing language model-based representations: (i) language modeling is unsupervised and information or metadata other than the raw sentences are not needed, which lets us leverage a large amount of available text on the web for training a powerful representation and (ii) language modeling is a general-purpose task, so a representation that is relevant to language modeling is also relevant for syntactic and semantic similarities, helping machines in NLP tasks (e.g. machine translation, parsing, part-of-speech tagging or information retrieval)

Inspired by this idea, in a previous work, we proposed distributed vector representations of biological sequence segments instead of $k$-mers, namely bio-vector for general use, and ProtVec for proteins. We used the skip-gram neural network for this purpose. Skipgram is a neural network with an objective analogous to a language modeling task (Bojanowski et al., 2016; Mikolov et al., 2013). However, instead of predicting the next word (or next $k$-mer) from the previous words, the task is to predict the surrounding words for a given word. We use large protein sequence databases (e.g. SwissProt) without any metadata for training a general-purpose representation of protein $k$-mers. The objective of the skip-gram neural network is to maximize the log-likelihood of observing the contexts of $k$-mers in a window of $N$ around it:

$$
\sum_{t=1}^{|\mathcal{C}|} \sum_{c \in[t-N, t+N]} \log p\left(w_{c} \mid w_{t}\right)
$$

where $w_{t}$ is the current $k$-mer and $c$ indicates the indices around index $t$ in the window size of $N$. $\mathcal{C}$ contains all existing $k$-mer contexts in the training data (e.g. all $k$-mer contexts that exist in SwissProt for all possible 3-mers). This likelihood is parameterized by $k$ mer representations $\left(v_{t}\right)$ and $k$-mer context representation $\left(v_{c}\right)$ in the skip-gram neural network:

$$
p\left(w_{c} \mid w_{t} ; \theta\right)=\frac{e^{v_{c} \cdot v_{t}}}{\sum_{c^{\prime} \in \mathcal{C}} e^{v_{c} \cdot v_{t}}} .
$$

Since including all existing contexts in the above-mentioned softmax function is computationally expensive, negative sampling is used during training. After training the $k$-mer representations, to represent a given protein sequence, we used summation embedding of the existing $k$-mers in the sequence. Such representations have proven helpful in protein function annotation tasks (Asgari and Mofrad, 2015; Zhou et al., 2019).

\subsection{ML methods}

After encoding the peptides as feature vectors, we used ML algorithms to classify the peptides as epitopes versus non-epitopes. For this binary classification, we used SVMs with the RBF kernel. All the algorithms were implemented by the Sklearn package in Python. SVMs have been used extensively in linear epitope prediction (BCPreds, LBTope, AAP, etc.) (Chen et al., 2007; El-Manzalawy et al., 2008; Singh et al., 2013) and they have also been applied for sequence-based prediction tasks (Leslie et al., 2002; Wu and Zhang, 2008; Zou et al., 2013). We used a grid search to optimize the parameters $C$ and $\gamma$ over the range [1000-0.0001], with steps of a power of two.

\subsection{Performance evaluation}

We used 5-fold cross-validation on the training dataset for optimizing the hyper-parameters of our model and reported the performance averaged over the held-out folds using common metrics for evaluating binary classification algorithms. Specifically, we calculated the prediction accuracy (ACC), precision (Precision), recall/ sensitivity $\left(S_{n}\right), F 1$ score $(F 1)$, Matthews correlation coefficient (MCC), and area under the receiver operating characteristic (ROC) curve $\left(R O C_{A U C}\right)$. If the dataset was unbalanced, we used balanced accuracy as the accuracy metric.

\section{Results}

Our first task was to identify features that will be included in our ML model. To this end, we selected the best performing features for our ML model using 5-fold cross-validation on the BCPreds dataset (Supplementary Section 2) and then trained the classifier with the selected features on three datasets: (i) EpitopeVec(BCPreds) trained on the BCPreds dataset; (ii) EpitopeVec(LBTope) trained on the LBTope dataset; and (iii) EpitopeVec(iBCE-EL) trained on the iBCE-EL training dataset. We then compared the performance of our method with previously published methods. In addition, we benchmarked the methods that were not tested on the selected benchmarking datasets used previously (e.g. BepiPred, BepiPred2.0, iBCE-EL, EpiDope and DLBEpitope). As there were many peptides in our test datasets that were exact matches $(100 \%$ homologous $)$ with peptides in DLBEpitope's training set, we retested DLBEpitope on the homology-reduced test sets as well (Supplementary Section 3). For the methods reporting per-residue scores (e.g. BepiPred, BepiPred2.0 and EpiDope), we averaged the scores for all constituent residues to obtain a single score for the peptide (Supplementary Section 3). 
Table 2. Comprehensive benchmark of linear BCE predictors on different datasets, comparing EpitopeVec with previously published methods for linear BCE prediction

\begin{tabular}{|c|c|c|c|c|c|c|c|c|}
\hline Method & ROC_AUC & Accuracy & Precision + & Precision- & Recall+ & Recall- & $F 1$ & MCC \\
\hline \multicolumn{9}{|l|}{ BCPreds set } \\
\hline BepiPred $^{\mathrm{a}}$ & 0.665 & 61.19 & 0.65 & 0.59 & 0.49 & 0.74 & 0.61 & 0.232 \\
\hline ABCPred ${ }^{\mathrm{b}}$ & 0.643 & - & - & - & - & - & - & - \\
\hline$A A P^{\mathrm{c}}$ & 0.7 & 64.05 & - & - & - & - & - & - \\
\hline BCPreds ${ }^{\mathrm{d}}$ & 0.758 & 67.9 & - & - & 0.73 & 0.63 & - & 0.360 \\
\hline Cobe - Pro $^{e}$ & 0.768 & 71.4 & - & - & - & - & - & - \\
\hline LBTope $e^{\mathrm{f}}$ & - & 51.57 & - & - & - & - & - & - \\
\hline$i B C E-E L^{g}$ & 0.576 & 48.71 & 0.49 & 0.15 & 0.97 & 0.01 & 0.33 & -0.009 \\
\hline BepiPred $2.0^{h}$ & 0.432 & 49.78 & 0.49 & 0.5 & 0.11 & 0.89 & 0.41 & -0.006 \\
\hline DLBEpitope $e^{\mathrm{i}}$ & 0.647 & 59.62 & 0.72 & 0.56 & 0.32 & 0.88 & 0.56 & 0.232 \\
\hline DLBEpitope $e^{m}$ & 0.515 & 50.78 & 0.4 & 0.63 & 0.14 & 0.88 & 0.47 & 0.023 \\
\hline EpiDope $e^{j}$ & 0.575 & 50.71 & 0.81 & 0.5 & 0.02 & 1 & 0.35 & 0.067 \\
\hline EpitopeVec(BCPreds) $)^{l}$ & 0.889 & 81.31 & 0.816 & 0.811 & 0.807 & 0.819 & 0.811 & 0.627 \\
\hline EpitopeVec(LBTope) & 0.645 & 57.13 & 0.56 & 0.59 & 0.69 & 0.45 & 0.57 & 0.147 \\
\hline EpitopeVec(iBCE - EL) & 0.596 & 57.48 & 0.57 & 0.57 & 0.57 & 0.57 & 0.57 & 0.15 \\
\hline \multicolumn{9}{|l|}{ Chen set } \\
\hline BepiPred & 0.665 & 60.44 & 0.64 & 0.58 & 0.47 & 0.74 & 0.6 & 0.217 \\
\hline$A A P^{l}$ & 0.7 & 71.09 & - & - & 0.61 & 0.75 & - & 0.366 \\
\hline$A A P+$ scales $^{c}$ & - & 72.54 & - & - & 0.64 & 0.76 & - & 0.404 \\
\hline APCPred ${ }^{\mathrm{k}}$ & 0.809 & 72.94 & - & - & 0.70 & 0.76 & - & 0.460 \\
\hline Cobe-Pro & 0.829 & 78 & - & - & - & - & - & - \\
\hline LBTope & - & 53.33 & - & - & - & - & - & - \\
\hline$i B C E-E L$ & 0.528 & 49.4 & 0.5 & 0.4 & 0.96 & 0.02 & 0.35 & -0.036 \\
\hline BepiPred2.0 & 0.424 & 49.31 & 0.47 & 0.5 & 0.11 & 0.88 & 0.4 & -0.021 \\
\hline DLBEpitope & 0.654 & 61.45 & 0.75 & 0.57 & 0.35 & 0.88 & 0.58 & 0.272 \\
\hline DLBEpitope $e^{m}$ & 0.535 & 51.81 & 0.38 & 0.69 & 0.15 & 0.88 & 0.50 & 0.050 \\
\hline EpiDope & 0.559 & 50.68 & 0.77 & 0.5 & 0.02 & 0.99 & 0.35 & 0.061 \\
\hline EpitopeVec(BCPreds) & 0.958 & 88.30 & 0.85 & 0.92 & 0.93 & 0.83 & 0.88 & 0.770 \\
\hline EpitopeVec(LBTope) & 0.658 & 57.91 & 0.56 & 0.61 & 0.71 & 0.44 & 0.57 & 0.164 \\
\hline EpitopeVec $(i B C E-E L)$ & 0.589 & 56.70 & 0.57 & 0.57 & 0.57 & 0.57 & 0.57 & 0.134 \\
\hline \multicolumn{9}{|l|}{ ABCPred set } \\
\hline BepiPred & 0.624 & 57.71 & 0.6 & 0.56 & 0.46 & 0.69 & 0.57 & 0.158 \\
\hline$A A P$ & 0.782 & 73.14 & - & - & 0.50 & 0.96 & - & 0.518 \\
\hline APCPred & 0.794 & 73.00 & - & - & 0.65 & 0.81 & - & 0.466 \\
\hline ABCPred ${ }^{l}$ & - & 65.93 & - & - & 0.67 & 0.65 & - & 0.319 \\
\hline BCPreds & 0.801 & 74.57 & - & - & 0.70 & 0.79 & - & 0.493 \\
\hline LBTope & - & 57.90 & - & - & - & - & - & - \\
\hline$i B C E-E L$ & 0.588 & 52.7 & 0.51 & 0.72 & 0.96 & 0.09 & 0.42 & 0.112 \\
\hline BepiPred 2.0 & 0.399 & 49.28 & 0.39 & 0.5 & 0.02 & 0.96 & 0.35 & -0.04 \\
\hline DLBEpitope & 0.7 & 63.86 & 0.78 & 0.59 & 0.39 & 0.89 & 0.61 & 0.319 \\
\hline DLBEpitope $e^{m}$ & 0.567 & 52.79 & 0.43 & 0.68 & 0.17 & 0.89 & 0.51 & 0.078 \\
\hline EpiDope & 0.599 & 50.64 & 0.76 & 0.5 & 0.02 & 0.99 & 0.35 & 0.059 \\
\hline EpitopeVec(BCPreds) & 0.929 & 85.64 & 0.84 & 0.88 & 0.89 & 0.83 & 0.86 & 0.714 \\
\hline EpitopeVec(LBTope) & 0.723 & 64.79 & 0.62 & 0.69 & 0.75 & 0.54 & 0.64 & 0.303 \\
\hline EpitopeVec $(i B C E-E L)$ & 0.616 & 59.28 & 0.68 & 0.56 & 0.35 & 0.83 & 0.57 & 0.212 \\
\hline \multicolumn{9}{|l|}{ Blind387 set } \\
\hline BepiPred & 0.627 & 56.59 & 0.55 & 0.58 & 0.53 & 0.6 & 0.57 & 0.132 \\
\hline$A A P$ & 0.689 & 64.60 & - & - & 0.64 & 0.65 & - & 0.292 \\
\hline ABCPred & - & 66.41 & - & - & 0.72 & 0.62 & - & - \\
\hline BCPreds & 0.699 & 65.89 & & & 0.66 & 0.66 & & 0.318 \\
\hline$i B C E-E L$ & 0.501 & 43.4 & 0.44 & 0.17 & 0.84 & 0.03 & 0.32 & -0.227 \\
\hline BepiPred 2.0 & 0.62 & 55.53 & 0.75 & 0.55 & 0.16 & 0.95 & 0.48 & 0.181 \\
\hline DLBEpitope & 0.649 & 59.72 & 0.66 & 0.70 & 0.27 & 0.92 & 0.59 & 0.264 \\
\hline DLBEpitope $e^{m}$ & 0.555 & 52.67 & 0.29 & 0.82 & 0.13 & 0.92 & 0.52 & 0.075 \\
\hline EpiDope & 0.541 & 50.80 & 1 & 0.52 & 0.02 & 1 & 0.36 & 0.091 \\
\hline EpitopeVec(BCPreds) & 0.778 & 71.83 & 0.75 & 0.7 & 0.63 & 0.81 & 0.72 & 0.445 \\
\hline EpitopeVec(LBTope) & 0.756 & 65.55 & 0.60 & 0.75 & 0.82 & 0.49 & 0.64 & 0.329 \\
\hline EpitopeVec $(i B C E-E L)$ & 0.724 & 68.65 & 0.73 & 0.67 & 0.57 & 0.80 & 0.68 & 0.384 \\
\hline \multicolumn{9}{|l|}{ LBtope set } \\
\hline BepiPred & 0.566 & 54.57 & 0.55 & 0.54 & 0.49 & 0.61 & 0.55 & 0.092 \\
\hline LBTope ${ }^{l}$ & 0.69 & 64.86 & - & - & - & - & - & - \\
\hline BCPreds & - & 52.56 & - & - & - & - & - & - \\
\hline
\end{tabular}


Table 2. (continued)

\begin{tabular}{|c|c|c|c|c|c|c|c|c|}
\hline Method & ROC_AUC & Accuracy & Precision+ & Precision- & Recall+ & Recall- & $F 1$ & MCC \\
\hline$i B C E-E L$ & 0.619 & 52.2 & 0.51 & 0.9 & 0.99 & 0.05 & 0.39 & 0.135 \\
\hline BepiPred 2.0 & 0.476 & 49.95 & 0.5 & 0.5 & 0.13 & 0.87 & 0.42 & -0.001 \\
\hline DLBEpitope & 0.769 & 67.27 & 0.82 & 0.62 & 0.44 & 0.9 & 0.65 & 0.389 \\
\hline DLBEpitope ${ }^{m}$ & 0.504 & 50.89 & 0.60 & 0.43 & 0.15 & 0.87 & 0.41 & 0.025 \\
\hline EpiDope & 0.559 & 50.34 & 0.68 & 0.5 & 0.01 & 0.99 & 0.35 & 0.036 \\
\hline EpitopeVec(BCPreds) & 0.548 & 52.98 & 0.55 & 0.52 & 0.32 & 0.74 & 0.51 & 0.065 \\
\hline EpitopeVec(LBTope $)^{l}$ & 0.838 & 75.62 & 0.75 & 0.75 & 0.76 & 0.75 & 0.76 & 0.512 \\
\hline EpitopeVec(iBCE - EL) & 0.602 & 57.12 & 0.56 & 0.58 & 0.62 & 0.53 & 0.57 & 0.143 \\
\hline \multicolumn{9}{|l|}{ iBCE-EL training set } \\
\hline BepiPred & 0.556 & 53.76 & 0.49 & 0.59 & 0.47 & 0.61 & 0.54 & 0.076 \\
\hline$i B C E-E L^{l}$ & 0.782 & 72.9 & - & - & 0.716 & 0.739 & - & 0.454 \\
\hline BepiPred 2.0 & 0.51 & 50.99 & 0.55 & 0.56 & 0.06 & 0.96 & 0.41 & 0.045 \\
\hline EpiDope & 0.582 & 50.57 & 0.8 & 0.56 & 0.01 & 1 & 0.37 & 0.064 \\
\hline EpitopeVec(BCPreds) & 0.555 & 53.78 & 0.52 & 0.58 & 0.31 & 0.76 & 0.52 & 0.085 \\
\hline EpitopeVec(LBTope) & 0.718 & 63.41 & 0.56 & 0.71 & 0.73 & 0.54 & 0.62 & 0.270 \\
\hline EpitopeVec $(i B C E-E L)^{l}$ & 0.789 & 71.4 & 0.7 & 0.73 & 0.64 & 0.77 & 0.71 & 0.419 \\
\hline \multicolumn{9}{|l|}{ iBCE-EL ind. set } \\
\hline BepiPred & 0.568 & 55.17 & 0.5 & 0.6 & 0.47 & 0.63 & 0.55 & 0.104 \\
\hline$i B C E-E L$ & 0.786 & 73.40 & 0.66 & 0.8 & 0.79 & 0.68 & 0.73 & 0.454 \\
\hline BepiPred 2.0 & 0.486 & 51.62 & 0.56 & 0.57 & 0.08 & 0.95 & 0.43 & 0.065 \\
\hline EpiDope & 0.595 & 50.47 & 0.7 & 0.56 & 0.01 & 1 & 0.37 & 0.049 \\
\hline EpitopeVec(BCPreds) & 0.571 & 54.20 & 0.52 & 0.59 & 0.31 & 0.78 & 0.53 & 0.095 \\
\hline EpitopeVec(LBTope $)$ & 0.745 & 65.43 & 0.57 & 0.74 & 0.76 & 0.55 & 0.64 & 0.311 \\
\hline EpitopeVec $(i B C E-E L)$ & 0.782 & 70.69 & 0.68 & 0.73 & 0.63 & 0.76 & 0.7 & 0.402 \\
\hline \multicolumn{9}{|l|}{ Viral method } \\
\hline EpitopeVec-viral (on viral training) ${ }^{l}$ & 0.875 & 79.73 & 0.718 & 0.843 & 0.698 & 0.67 & 0.850 & 0.554 \\
\hline EpitopeVec-viral on BCPreds viral & 0.756 & 72.02 & 0.968 & 0.190 & 0.648 & 0.793 & 0.541 & 0.264 \\
\hline EpitopeVec-viral on BCPreds bacterial & 0.569 & 54.07 & 0.301 & 0.770 & 0.378 & 0.704 & 0.535 & 0.076 \\
\hline EpitopeVec-viral on BCPreds eukaryotic & 0.512 & 52.26 & 0.384 & 0.663 & 0.357 & 0.689 & 0.52 & 0.046 \\
\hline$i B C E-E L$ on $B C P r e d s$ viral & 0.509 & 50.16 & 0.93 & 0.08 & 0.97 & 0.03 & 0.5 & 0.005 \\
\hline$i B C E-E L$ on BCPreds bacterial & 0.574 & 50.14 & 0.25 & 1 & 1 & 0.003 & 0.2 & 0.027 \\
\hline$i B C E-E L$ on BCPreds eukaryotic & 0.571 & 47.48 & 0.348 & 0.18 & 0.94 & 0.006 & 0.26 & -0.155 \\
\hline
\end{tabular}

Note: The training dataset for EpitopeVec is indicated in parentheses. Rows with gray cells indicate cases where the results for the corresponding methods could not be recreated, and for which we could include only the scores for metrics reported in the original publication. The highest values per metric and test set are indicated in bold. ' + ' indicates epitope predictions and ' - ' indicates non-epitope predictions.

${ }^{\mathrm{a}}$ Larsen et al. (2006).

bSaha and Raghava (2006).

${ }^{\mathrm{c}}$ Chen et al. (2007).

${ }^{\mathrm{d}}$ El-Manzalawy et al. (2008).

${ }^{\mathrm{e}}$ Sweredoski and Baldi (2009).

${ }^{\mathrm{f}}$ Singh et al. (2013).

${ }^{g}$ Manavalan et al. (2018).

${ }^{\mathrm{h}}$ Jespersen et al. (2017).

${ }^{i}$ Liu et al. (2020).

iCollatz et al. (2021).

${ }^{\mathrm{k}}$ Shen et al. (2015).

${ }^{1}$ Averaged over the held-out folds of the 5-fold cross-validation.

${ }^{m}$ Tested after removing homologous sequences (peptides that were more than $80 \%$ homologous in comparison to the peptides in the training set) from the test set.

\subsection{Discriminative power of the protein features}

We used several feature sets in our ML models and investigated which feature set had the best discriminative power when used independently. We tested the SVM model on the BCPreds dataset with 5 -fold cross-validation including individual feature sets. All of the ML models were trained on the BCPreds dataset with 5-fold crossvalidation, $C$, and $\gamma$ parameters for the RBF kernel were optimized via a grid search through cross-validation, and performance was averaged over the held-out folds. Use of the chain-transition-distribution features (Dubchak et al., 1995) resulted in an accuracy of $61 \%$, whereas the AAC scales performed quite similarly, with their accuracy ranging from $63 \%$ (AAC) to $65 \%$ (DPC). With $k$-mer representations, higher values of $k$ resulted in a better accuracy (the highest being $69.9 \%$ with $k=4$ ) and the use of ProtVec features resulted in an accuracy of $70 \%$. Use of the AAP antigenicity scale resulted in an accuracy of $68.55 \%$ and use of the AAT antigenicity scale produced the highest accuracy of $78.67 \%$. When taking combinations of different feature sets, we achieved the highest accuracy of $81.31 \%$ by combining the composition-based features (AAP, AAT and AAC) with the sequence representation-based features (Protvec). This feature set was selected for use with our new method called EpitopeVec (Supplementary Section 2).

\subsection{Evaluation on the BCPreds dataset}

In the 5-fold cross-validation, our method [EpitopeVec(BCPreds)] performed the best with an average accuracy of $81.31 \%(13.41 \%$ higher than the original BCPreds method) (Table 2) and a ROC- 


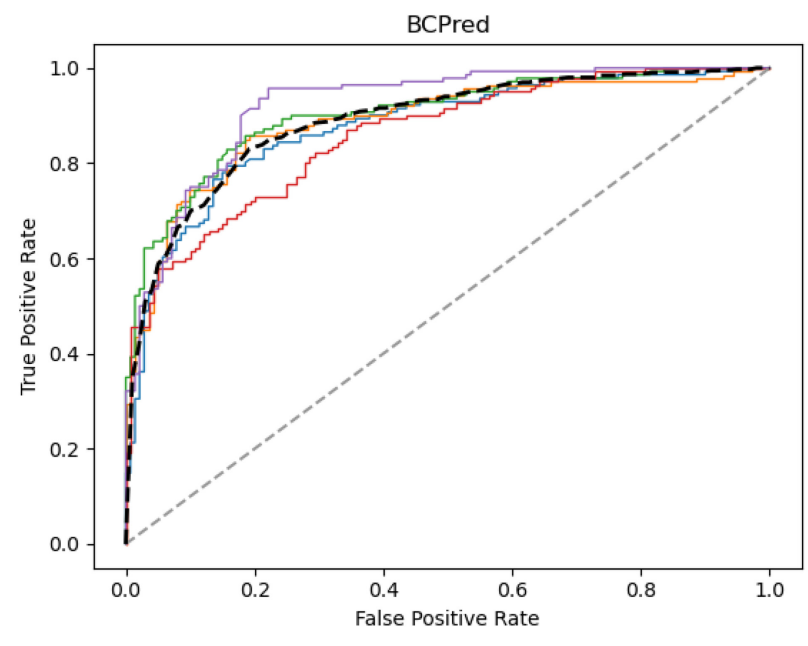

Fig. 1. ROC curve of epitope prediction in 5-fold cross-validation on the BCPreds dataset, where the mean is shown as a bold dashed line and the random performance $($ ROCAUC $=0.5)$ is shown as a gray dashed line. The five different colored lines represent the performance of each of the 5 -folds

AUC of 0.889 (Fig. 1). The EpitopeVec(LBtope) and EpitopeVec(iBCE-EL) models had an accuracy of $57.13 \%$ and $57.48 \%$ on the BCPreds dataset, respectively. In previously published work, the accuracy of the method trained on the IEDBderived datasets (LBtope) was reported to be $51.57 \%$ (Singh et al., 2013), while the accuracy of other methods trained on the Bcipepderived datasets (AAP, ABCPred and Cobe-Pro) was reported to be between $64 \%$ and $71 \%$ (Chen et al., 2007; Saha and Raghava, 2006; Sweredoski and Baldi, 2009). Among the methods that we benchmarked, iBCE-EL, BepiPred2.0 and EpiDope had the lowest performance on this dataset, with accuracies ranging from $49 \%$ to $51 \%$ (Table 2). BepiPred and DLBEpitope had slightly better accuracies $(61.2 \%$ and $59.6 \%)$, but the accuracy of DLBEpitope dropped to $51 \%$ after retesting on the homology-reduced BCPreds set (removing peptides that were $>80 \%$ homologous to DLBEpitope's training set).

\subsection{Evaluation on the Chen dataset}

The Chen method (AAP method) uses a scale specifically designed based on the assumption that certain AAPs are favored in the epitope regions. It was the first method to incorporate differences between epitopes and non-epitopes by making use of the AAP antigenicity scale as a discriminative feature. We achieved an accuracy of $88.30 \%$ (Table 2) with the EpitopeVec(BCPreds) model on this dataset, which is $10 \%$ higher than the next best method, CobePro $(78 \%)$ (Sweredoski and Baldi, 2009), and 16\% higher than the original AAP method $(71.09 \%)$ (Chen et al., 2007). This dataset was derived from the Bcipep database and was not reduced for homology, which explains why we observed a higher accuracy than that for the BCPreds dataset. The accuracy of EpitopeVec(LBTope) was $57.91 \%$ and EpitopeVec(iBCE-EL) was $56.70 \%$ on this dataset. The accuracy of the LBTope method (trained on IEDB-derived dataset) was reported to be $53.33 \%$ (Singh et al., 2013). The accuracies of the methods that we benchmarked (BepiPred2.0, iBCE-EL, BepiPred, EpiDope and DLBEpitope) ranged from $49.3 \%$ to $61.5 \%$, with DLBEpitope having the highest accuracy of $61.5 \%$, however, its accuracy dropped to $51.81 \%$ after retesting on the homologyreduced Chen set. Notably, we observed that the performance of most methods trained on the IEDB-derived sets was lower on this dataset.

\subsection{Evaluation on the ABCPred dataset}

The original ABCPred method is based on a recurrent neural network with an input vector of 16 residues and a sparse binary encoding, and it was one of the first methods to use machine learning for linear BCE prediction. The accuracy of EpitopeVec(BCPreds) on this dataset was $85.64 \%$, which is $20 \%$ higher than the original ABCPred method (65.93\%) (Saha and Raghava, 2006), and 11\% higher than BCPreds (74.57\%) (El-Manzalawy et al., 2008). As this dataset is also derived from the Bcipep database and was not specifically homology-reduced, we observed a higher accuracy compared to the BCPreds dataset. The accuracy of EpitopeVec(LBTope) was $64.79 \%$ and EpitopeVec(iBCE-EL) was $59.28 \%$ on the ABCPred dataset. The accuracy of methods, such as AAP and APCPred, was reported to be around 73\% (Chen et al., 2007; Shen et al., 2015), while that of LBTope was reported to be $57.90 \%$ (Singh et al., 2013).

We also tested EpitopeVec on the Blind387 set that was published along with ABCPred. This is an independent test set with only some of the peptides (59 out of 187) from the Bcipep database. EpitopeVec(BCPreds) obtained an accuracy of $71.83 \%$, outperforming the accuracies previously reported for AAP $(64.60 \%)$, ABCPred $(66.41 \%)$ and BCPreds (65.89\%) (Chen et al., 2007; El-Manzalawy et al., 2008; Saha and Raghava, 2006). The accuracies obtained by our benchmarked methods (BepiPred, iBCE-EL, EpiDope, DLBEpitope and BepiPred2.0) ranged between $43 \%$ and $60 \%$ (Table 2). The accuracies of Epitope(LBTope) and Epitope(iBCEEL) on the Blind 387 set were $65.55 \%$ and $68.65 \%$, respectively.

\subsection{Evaluation on the LBTope dataset}

LBtope is one of the first methods that made use of datasets compiled from the IEDB database. In 5-fold cross-validation on this dataset, our EpitopeVec(LBTope) model had an accuracy of $75.62 \%$ (Table 2), which was $11 \%$ higher than for the original LBTope method $(64.86 \%)$ (Singh, 2013). The performance of methods trained on Bciped-derived datasets [BCPreds, Epitope Vec(BCPreds)] dropped substantially on this dataset, with the accuracy of BCPreds being $52.56 \%$ (El-Manzalawy et al., 2008) and the accuracy of the EpitopeVec(BCPreds) model being $52.98 \%$. The accuracy of the EpitopeVec(iBCE-EL) model was $57.12 \%$. All other methods that we benchmarked (iBCE-EL, EpiDope, BepiPred and BepiPred2.0), except for DLBEpitope, had low performances on this dataset, with their accuracies ranging from $50 \%$ to $55 \%$. DLBEpitope had an accuracy of $67.2 \%$, but it dropped to $50.89 \%$ after retesting on the homology-reduced LBTope set.

\subsection{Evaluation on the iBCE-EL dataset}

The iBCE-EL method is an ensemble method that uses Gradient Boosting and an Extra tree classifier. In 5-fold cross-validation on the iBCE-EL training dataset, our EpitopeVec(iBCE-EL) model had an average accuracy of $71.4 \%$ (Table 2 ), which is slightly lower than that of the original iBCE-EL method $(73.40 \%)$. However, the average ROC_AUC of EpitopeVec(iBCE-EL) was 0.789 , which is marginally higher than that of the iBCE-EL method (0.782). On the iBCE-EL independent test set, the performance of EpitopeVec(iBCEEL) was slightly lower (accuracy: $70.69 \%$ and ROC_AUC: 0.782 ) than that of the iBCE-EL method (accuracy: $73.40 \%$ and ROC_AUC: 0.786). The accuracy for the methods that we benchmarked (BepiPred, BepiPred2.0 and EpiDope) ranged from $50 \%$ to $53 \%$ on the iBCE-EL training set and from $50 \%$ to $55 \%$ on the iBCE-EL independent set. The accuracies of EpitopeVec(BCPreds) and EpitopeVec(LBtope) were $53.78 \%$ and $63.41 \%$ on the iBCE-EL training set, while $54.20 \%$ and $65.43 \%$ on the iBCE-EL independent set.

\subsection{Cross-testing results}

In cross-testing on datasets different than their training sets, all of our three models had better performance than their respective original methods (Fig. 2) as well as other methods that we benchmarked (Table 2). However, we observed that when a method was trained on a dataset compiled from the Bcipep database, and then tested on a dataset compiled from the IEDB and vice versa, the performance dropped substantially. Our EpitopeVec(BCPreds) model had good performance ( $>80 \%$ accuracy) on other Bcipep-derived datasets (ABCPred, Chen), but the performance dropped 


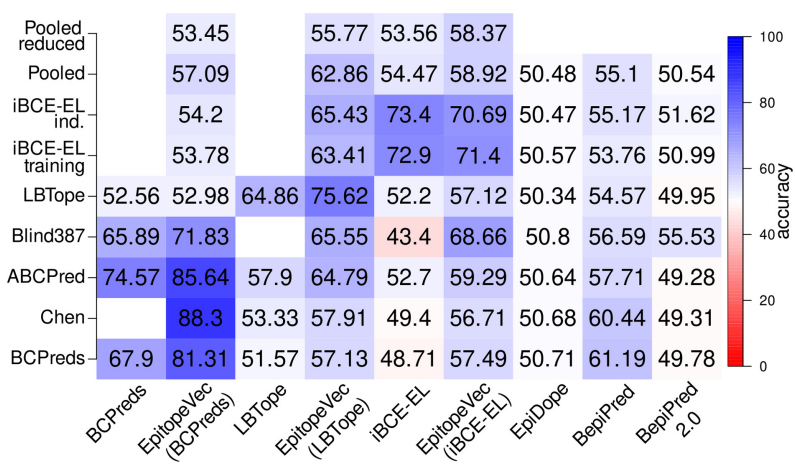

Fig. 2. Heatmap showing the cross-testing performance of EpitopeVec when trained and tested on different datasets and comparison with other methods ( $\mathrm{x}$-axis, testing method; y-axis testing data; for training and testing on the same dataset, the accuracy averaged over 5 -fold cross-validation is shown).

substantially when testing on the IEDB-derived datasets (LBTope, iBCE-EL). The same was true for BCPred, LBTope and the other methods as well. Therefore, to test the overall performance of the methods, we created three pooled datasets [one each for EpitopeVec(BCPreds), EpitopeVec(LBTope) and EpitopeVec(iBCEEL) models], where we combined all the testing sets except the respective training set of that model. We also created pooled-reduced sets, where we removed peptides in the pooled set that were homologous to the respective training set (inter-homology reduction) and similar sequences within the pooled sets (intra-homology reduction) using CD-HIT with a threshold of $80 \%$. The performance of the EpitopeVec(iBCE-EL) model (58.37\% accuracy, 0.615 AUC) was the best $(5 \%$ higher accuracy than iBCE-EL) on its pooled-reduced set and overall it had better generalizability. The differences in the performance of the EpitopeVec method when trained on different datasets show that the performance is dependent on the source of the training data (Fig. 2). The substantial performance difference, we observed between cross-testing and 5 -fold cross-validation on datasets derived from the Bcipep database and the IEDB indicates that most classifiers lack generalization, potentially caused by the fundamental differences in the nature of the underlying data (Odorico and Pellequer, 2003). When we computed the Pearson correlation of the AAT scale (the most discriminative feature in our method) derived from the Bcipep sets and the IEDB sets, it was relatively weak (0.41), indicating large differences in the composition of the epitopic peptides from both the databases.

\subsection{Improving epitope prediction accuracy with domain- specific datasets}

In light of the observed lack of generalization of most methods and only slightly better generalizability of our method, we created a linear BCE predictor specific to viral peptides. When analyzing the composition of the Bcipep and IEDB databases, we found that Bcipep had a strong bias toward viruses, with $80 \%$ of the peptides originating from viral antigens. To investigate this further, we created a dataset of viral epitopes with experimentally verified positive and negative epitopes from the IEDB dataset and trained our model on this dataset with EpitopeVec. We cross-tested this new viral predictor (EpitopeVec-viral) on the homology-reduced BCPreds dataset (peptides in the BCPreds set that were more than $80 \%$ homologous to peptides in our training viral set were removed). The BCPreds dataset is composed of antigens from different species (i.e. viral, bacterial and eukaryotic), therefore, we were interested in the specific performance on antigens originating from different species. The performance of EpitopeVec-viral on viral peptides $(72.02 \%$ accuracy, 0.756 ROC_AUC) was substantially higher than that on bacterial (54.07\% accuracy, 0.569 ROC_AUC) and eukaryotic $(52.26 \%$ accuracy, 0.512 ROC_AUC) peptides (Table 2 and Fig. 3a). The

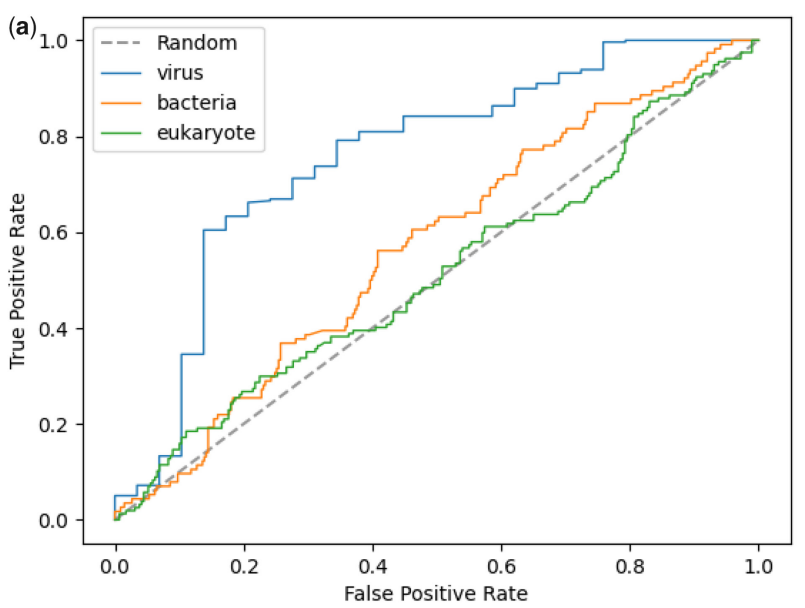

EpitopeVec-viral

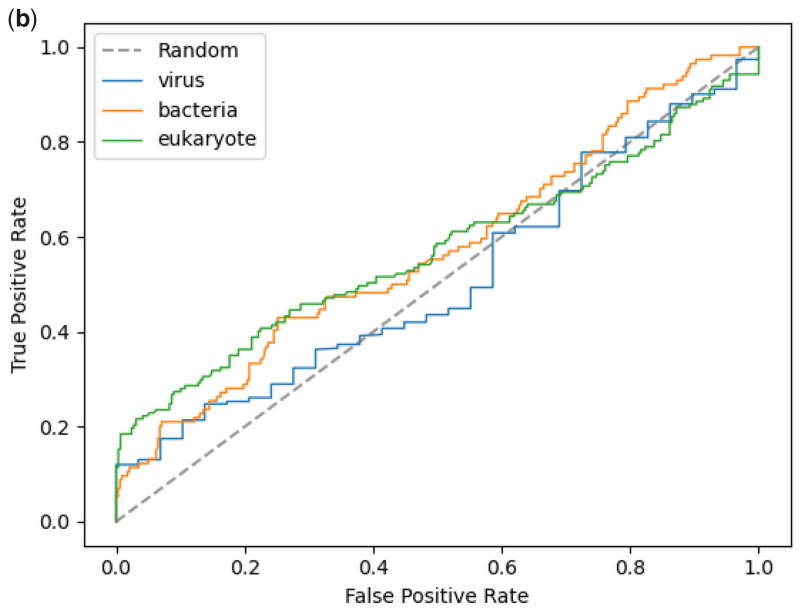

iBCE-EL

Fig. 3. Area under the ROC curve for each antigen type in the BCPreds set, where the (a) EpitopeVec-viral predictor and (b) iBCE-EL were used as the predictive models

performance of iBCE-EL on domain-specific peptides was substantially below that of EpitopeVec-viral (Table 2 and Fig. 3 b).

\subsection{Predicting epitopes for SARS-CoV-1 and SARS-CoV- \\ 2 viruses}

We tested our viral predictor on a set of 10 linear epitope peptides compiled in Grifoni et al. (2020) for SARS-CoV-1 from experimentally verified epitopes in the IEDB. We successfully predicted 7 out of 10 peptides as epitopes for SARS-CoV-1 with our EpitopeVecviral model. We also compiled a set of experimentally verified epitopes and non-epitopes for SARS-CoV-2 from the IEDB and tested our viral predictor on these peptides. We predicted 7 out of 9 epitopes and 10 out of 10 non-epitopes correctly (Supplementary Section 4). Our viral predictor has very high precision in predicting both experimentally validated epitopes and non-epitopes derived from independent datasets.

\section{Discussion and conclusions}

In this work, we describe a ML framework for predicting linear BCEs from a combination of amino acid features, modified antigenicity scales (AAT) and context-independent protein embeddings (ProtVec). 


\subsection{Comprehensive benchmarking}

We performed a comprehensive benchmarking of different linear BCE prediction methods over the existing BCE datasets.

This benchmarking introduces a meaningful comparison of existing methods in the literature by following the standard ML practices. In our benchmarking, we attempted to include all the accessible published methods, including methods that had not been previously tested across all our test datasets. The performance of most IEDBtrained methods dropped substantially when testing on Bcipepderived datasets and vice versa. Of the per-residue methods, the performance of EpiDope and BepiPred2.0 was close to random. Of these methods, though the overall performance was still low, BepiPred performed the best, which may explain why it is still extensively used (Raoufi et al., 2020).

\subsection{EpitopeVec performance}

We trained our method on three different datasets (BCPreds, LBTOPE and iBCE-EL). The EpitopeVec(BCPreds) and EpitopeVec(LBTope) models showed improved performance in 5fold cross-validation on their training sets in comparison to their respective original methods (BCPreds and LBTOPE). The EpitopeVec(iBCE-EL) model had a slightly lower performance compared to the iBCE-EL method in the 5 -fold cross-validation on the iBCE-EL training set and independent testing on the iBCE-EL independent set. We believe that this is because the original iBCE-EL method is an ensemble method using multiple ML algorithms. Overall, our EpitopeVec model showed better performance in crossvalidation, demonstrating that our method is superior to the stateof-the-art approaches in predicting linear BCEs.

\subsection{Generalizability and cross-testing}

In cross-testing on datasets, different than the training sets, the performance of our three models [EpitopeVec(BCPreds), EpitopeVec(LBTope) and EpitopeVec(iBCE-EL)] were not only better than the performances of the original methods (BCPreds, LBTope and iBCE-EL) but also than those of the recent methods (DLBEpitope, BepiPred2.0 and EpiDope) that we benchmarked. When testing on the pooled/pooled-reduced test sets, the performance of our method was also better than that of other methods, which shows a better generalization ability. However, we observed that in cross-testing, the performance of most methods dropped substantially, a known problem to ML approaches (Ng, 1997). In the case of BCE prediction, the relatively reduced performance in crosstesting was caused by the different compositions of residues in the positive and negative sets selected from these datasets. This indicates that properties distinguishing epitopic and non-epitopic peptides could be specific to the source of the antigen species (e.g. viral, bacterial or fungal antigens), as discussed previously (Gupta et al., 2013; Kozlova et al., 2018; Luštrek et al., 2013) and that creating a general-purpose classifier is difficult. To partially overcome the difficulties in creating a general-purpose linear BCE predictor, we and others (Gupta et al., 2013; Kozlova et al., 2018; Soria-Guerra et al., 2015) recommend creating an accurate, specialized predictor for specific antigenic types. We trained a linear viral BCE predictor on a viral dataset separately in favor of this conclusion. Upon crosstesting on the BCPreds dataset, the viral predictor performed substantially better on viral peptides than on bacterial and eukaryotic peptides.

\subsection{Applications and purposes}

Although almost $90 \%$ of BCEs are conformational in nature (Barlow et al., 1986), linear BCEs are widely used for profiling antibody signatures (Noya et al., 2005), epitope mapping (peptide microarray-based experiments) (Dudek et al., 2010; Potocnakova et al., 2016) and other fields in immunology (Hancock and O'Reilly, 2005). Additionally, previously published methods for predicting linear BCEs have been used for designing peptide-based vaccines (Chen et al., 2020; María et al., 2017; Nezafat et al., 2016; SoriaGuerra et al., 2015). To overcome limitations in applications as such, we proposed and proved that dedicated predictors, such as EpitopeVec-viral, are able to successfully identify experimentally validated SARS-CoV-1 and SARS-CoV-2 epitopes. Thus, our predictive model can be used on viral proteins and aid in designing new peptide-based vaccines (Grifoni et al., 2020; Malonis et al., 2020). We believe that improving linear BCE predictions will be of value to the research community, especially with generalized and pre-trained viral classifiers.

\subsection{Future direction}

For future development, large, non-redundant and experimentally well-characterized datasets could be compiled and standardized for the training and the evaluation of linear BCE predictive models. Lastly, we see linear epitope prediction as a stepping stone to the conformational problem and it will ease the process of developing conformational predictions because of its comparatively less complex nature.

\section{Acknowledgements}

We thank Tzu-Hao Kuo for commenting and help with testing the code, as well as Megan Foster for editing the text.

\section{Funding}

This work was supported by the Deutsches Zentrum für Infektionsforschung (DZIF, German Center for Infection Research) grant 'TI 06.901 - FP2016: Bioinformatics support for the development of a prophylactic HCV vaccine candidate' and Deutsche Forschungsgemeinschaft (DFG, German Research Foundation) under Germany's Excellence Strategy-EXC 2155Projektnummer 390874280.

Conflict of Interest: None declared.

\section{References}

Alipanahi,B. et al. (2015) Predicting the sequence specificities of DNA-and RNA-binding proteins by deep learning. Nat. Biotechnol., 33, 831-838.

Alix,A.J. (1999) Predictive estimation of protein linear epitopes by using the program PEOPLE. Vaccine, 18, 311-314.

Asgari,E. (2019) Life language processing: deep learning-based language-agnostic processing of proteomics, genomics/metagenomics, and human languages. PhD Thesis, UC Berkeley.

Asgari,E. and Mofrad,M.R. (2015) Continuous distributed representation of biological sequences for deep proteomics and genomics. PLoS One, 10, e0141287.

Asgari,E. et al. (2018) MicroPheno: predicting environments and host phenotypes from $16 \mathrm{~S}$ rRNA gene sequencing using a k-mer based representation of shallow sub-samples. Bioinformatics, 34, i32-i42.

Asgari,E. et al. (2019a) DeepPrime2Sec: deep learning for protein secondary structure prediction from the primary sequences. bttps: //www.biorxiv.org/content/10.1101/705426v1.

Asgari,E. et al. (2019b) Probabilistic variable-length segmentation of protein sequences for discriminative motif discovery (DiMotif) and sequence embedding (ProtVecX). Sci. Rep., 9, 1-16.

Awazu,A. (2017) Prediction of nucleosome positioning by the incorporation of frequencies and distributions of three different nucleotide segment lengths into a general pseudo k-tuple nucleotide composition. Bioinformatics, 33, 42-48.

Bairoch,A. (2000) The SWISS-PROT protein sequence database and its supplement TrEMBL in 2000. Nucleic Acids Res., 28, 45-48.

Barlow,D.J. et al. (1986) Continuous and discontinuous protein antigenic determinants. Nature, 322, 747-748.

Blythe,M.J. and Flower,D.R. (2005) Benchmarking B cell epitope prediction: underperformance of existing methods. Protein Sci., 14, 246-248.

Bojanowski,P. et al. (2016) Enriching word vectors with subword information. https://arxiv.org/abs/1607.04606v2.

Bonetta,R. and Valentino,G. (2020) Machine learning techniques for protein function prediction. Proteins Struct. Funct. Bioinforma., 88, 397-413.

Bryson,C.J. et al. (2010) Prediction of immunogenicity of therapeutic proteins: validity of computational tools. BioDrugs, 24, 1-8. 
Chen,H.Z. et al. (2020) Bioinformatics analysis of epitope-based vaccine design against the novel SARS-CoV-2. Infect. Dis. Poverty, 9, 88.

Chen,J. et al. (2007) Prediction of linear B-cell epitopes using amino acid pair antigenicity scale. Amino Acids, 33, 423-428.

Collatz,M. et al. (2021) EpiDope: a deep neural network for linear B-cell epitope prediction. Bioinformatics, 37, 448-455.

Dubchak,I. et al. (1995) Prediction of protein folding class using global description of amino acid sequence. Proc. Natl. Acad. Sci. USA, 92, 8700-8704.

Dudek,N.L. et al. (2010) Epitope discovery and their use in peptide based vaccines. Curr. Pharm. Des., 16, 3149-3157.

El-Manzalawy,Y. et al. (2008) Predicting linear B-cell epitopes using string kernels. J. Mol. Recognit., 21, 243-255.

Emini,E.A. et al. (1985) Induction of hepatitis A virus-neutralizing antibody by a virus-specific synthetic peptide. J. Virol., 55, 836-839.

Flower,D.R. (2007) Immunoinformatics. Predicting Immunogenicity In Silico. Preface. Springer

Galanis,K.A. et al. (2021) Linear B-cell epitope prediction for in silico vaccine design: A performance review of methods available via command-line interface. Int. J. Mol. Sci., 22, 1-19.

Giancarlo,R. et al. (2015) Epigenomic k-mer dictionaries: shedding light on how sequence composition influences in vivo nucleosome positioning. Bioinformatics, 31, 2939-2946.

Grabherr,M.G. et al. (2011) Full-length transcriptome assembly from RNA-seq data without a reference genome. Nat. Biotechnol., 29, 644-652.

Grifoni,A. et al. (2020) A sequence homology and bioinformatic approach can predict candidate targets for immune responses to SARS-CoV-2. Cell Host Microbe, 27, 671-680.e2.

Gupta,S. et al.; Open Source Drug Discovery Consortium. (2013) Identification of B-cell epitopes in an antigen for inducing specific class of antibodies. Biol. Direct, 8, 27.

Hancock,D.C. and O'Reilly,N.J. (2005) Synthetic peptides as antigens for antibody production. Methods Mol. Biol., 1474, 25-47.

Huang,Y. et al. (2010) CD-HIT Suite: a web server for clustering and comparing biological sequences. Bioinformatics, 26, 680-682.

Janeway,C. (2012) Immunobiology. 8th ednNew York: Garland Science.

Jespersen,M.C. et al. (2017) BepiPred-2.0: improving sequence-based B-cell epitope prediction using conformational epitopes. Nucleic Acids Res., 45, W24-W29.

Jolma,A. et al. (2013) DNA-binding specificities of human transcription factors. Cell, 152, 327-339.

Karplus,P.A. and Schulz,G.E. (1985) Prediction of chain flexibility in proteins - a tool for the selection of peptide antigens. Naturwissenschaften, 72, 212-213.

Kolaskar,A.S. and Tongaonkar,P.C. (1990) A semi-empirical method for prediction of antigenic determinants on protein antigens. FEBS Lett., 276, 172-174.

Kozlova,E.E.G. et al. (2018) Computational B-cell epitope identification and production of neutralizing murine antibodies against Atroxlysin-I. Sci. Rep., 8,14904 .

Larsen,J. et al. (2006) Improved method for predicting linear B-cell epitopes. Immunome Res., $2,2$.

Leslie,C. et al. (2002) The spectrum kernel: a string kernel for SVM protein classification. Pac. Symp. Biocomput., Lihue, Hawaii, 564-575.

Levitt,M. (1976) A simplified representation of protein conformations for rapid simulation of protein folding. J. Mol. Biol., 104, 59-107.

$\mathrm{Li}, \mathrm{Z}$. and Yu,Y. (2016) Protein secondary structure prediction using cascaded convolutional and recurrent neural networks. In: IJCAI International Joint Conference on Artificial Intelligence 9-15 July 2016. New York, NY, USA.

Liu,T. et al. (2020) Deep learning methods improve linear B-cell epitope prediction. BioData Min., 13, 1.

Liu,Y. et al. (2018) Learning structural motif representations for efficient protein structure search. Bioinformatics, 34, i773-i780.

Luštrek,M. et al. (2013) Epitope predictions indicate the presence of two distinct types of epitope-antibody-reactivities determined by epitope profiling of intravenous immunoglobulins. PLoS One, 8, e78605.
Malonis,R.J. et al. (2020) Peptide-based vaccines: current progress and future challenges. Chem. Rev., 120, 3210-3229.

Manavalan,B. et al. (2018) iBCE-EL: a new ensemble learning framework for improved linear B-cell epitope prediction. Front. Immunol., 9, 1695.

María,R.R. et al. (2017) The impact of bioinformatics on vaccine design and development. In, Vaccines. InTech.https://www.intechopen.com/books/vac cines/the-impact-of-bioinformatics-on-vaccine-design-and-development

Mikolov,T. et al. (2013) Distributed representations of words and phrases and their compositionality. In: Advances in Neural Information Processing Systems. Lake Tahoe, Nevada, pp. 3111-3119.

Nezafat,N. et al. (2016) Designing an efficient multi-epitope peptide vaccine against Vibrio cholerae via combined immunoinformatics and protein interaction based approaches. Comput. Biol. Chem., 62, 82-95.

Ng,A.Y. (1997) Preventing "overfitting" of cross-validation data. In: ICML. Nashville, Tennessee, USA.

Noya,O. et al. (2005) Immunodiagnosis of parasitic diseases with synthetic peptides. Curr. Protein Pept. Sci., 4, 299-308.

Odorico,M. and Pellequer,J.L. (2003) BEPITOPE: predicting the location of continuous epitopes and patterns in proteins. J. Mol. Recognit., 16, 20-22.

Pellequer,J.L. and Westhof,E. (1993) PREDITOP: a program for antigenicity prediction. J. Mol. Graph., 11, 204-210.

Pellequer,J.L. et al. (1991) Predicting location of continuous epitopes in proteins from their primary structures. Methods Enzymol., 203, 176-201.

Potocnakova,L. et al. (2016) An introduction to B-cell epitope mapping and in silico epitope prediction. J. Immunol. Res., 2016, 1-11.

Raoufi,E. et al. (2020) Epitope prediction by novel immunoinformatics approach: a state-of-the-art review. Int. J. Pept. Res. Ther., 26, 1155-1163.

Saha,S. and Raghava,G.P. (2006) Prediction of continuous B-cell epitopes in an antigen using recurrent neural network. Proteins, 65, 40-48.

Saha,S. et al. (2005) Bcipep: a database of B-cell epitopes. BMC Genomics, 6, 79.

Sanchez-Trincado,J.L. et al. (2017) Fundamentals and methods for T- and B-cell epitope prediction. J. Immunol. Res., 2017, 1-14.

Shen,W. et al. (2015) Predicting linear B-cell epitopes using amino acid anchoring pair composition. BioData Min., 8, 14.

Singh,H. et al. (2013) Improved method for linear B-cell epitope prediction using antigen's primary sequence. $\mathrm{PLOS} \mathrm{One}, 8, \mathrm{e} 62216$.

Soria-Guerra,R.E. et al. (2015) An overview of bioinformatics tools for epitope prediction: implications on vaccine development. J. Biomed. Inform., $53,405-414$

Sun,P. et al. (2018) Advances in in-silico B-cell epitope prediction. Curr. Top. Med. Chem., 19, 105-115.

Sweredoski,M.J. and Baldi,P. (2009) COBEpro: a novel system for predicting continuous B-cell epitopes. Protein Eng. Des. Sel., 22, 113-120.

Vita,R. et al. (2010) The immune epitope database 2.0. Nucleic Acids Res., 38, D854-D862.

Wood,D.E. and Salzberg,S.L. (2014) Kraken: ultrafast metagenomic sequence classification using exact alignments. Genome Biol., 15, R46.

Wu,S. and Zhang,Y. (2008) A comprehensive assessment of sequence-based and template-based methods for protein contact prediction. Bioinformatics, 24, 924-931.

Yang,X. and Yu,X. (2009) An introduction to epitope prediction methods and software. Rev. Med. Virol., 19, 77-96.

Yao,B. et al. (2012) SVMTriP: a method to predict antigenic epitopes using support vector machine to integrate tri-peptide similarity and propensity. PLoS One, 7, e45152.

Zhou,G. et al. (2020) Mutation effect estimation on protein-protein interactions using deep contextualized representation learning. NAR Genom. Bioinform., 2, iqaa015.

Zhou,N. et al. (2019) The CAFA challenge reports improved protein function prediction and new functional annotations for hundreds of genes through experimental screens. Genome Biol., 20, 1-23.

Zou,C. et al. (2013) An improved sequence based prediction protocol for DNA-binding proteins using SVM and comprehensive feature analysis. BMC Bioinformatics, 14, 90. 